\title{
Pyroprinting Sensitivity Analysis on the GPU
}

\author{
Douglas Brandt, Aldrin Montana, Bob Somers \\ Alex Dekhtyar, Chris Lupo \\ Computer Science Department \\ California Polytechnic State University \\ San Luis Obispo, United States \\ \{djbrandt, amontana, rsomers, dekhtyar, clupo\}@calpoly.edu
}

\author{
Michael Black, Anya Goodman, Chris Kitts \\ Biology Department \\ California Polytechnic State University \\ San Luis Obispo, United States \\ \{mblack, agoodman, ckitts\}@calpoly.edu
}

Keywords-CUDA, GPGPU, pyrosequence, pyrogram

\section{INTRODUCTION}

Microbial Source Tracking (MST) is a field in which microbial strains are identified and associated with a specific host source [1], [2], [3] (e.g., human, canine, avian, etc). Identifying the hosts of microbial strains lies at the heart of many studies of bacterial contamination in the environment. Being able to determine which host species is responsible, e.g., for fecal contamination of a creek, allows the parties involved to develop specific measures for addressing the contamination.

To address problems of strain differentiation that current MST methods [1], [2], [4] have, our research group has developed a novel pyrosequencing-based MST method [5] called pyroprinting. Pyrosequencing is an inexpensive DNA sequencing technique in which a complementary strand is synthesized for a given single-strand DNA fragment. Pyroprinting is the simultaneous pyrosequencing of multiple, possibly different, DNA fragments from the same organism. The resulting pattern of emitted light intensities, called a pyroprint, is used as a digital fingerprint of the organism. Pyroprints are generated from known highly variable regions, called intergenic transcribed spacers (ITS), located in each copy of the ribosomal RNA operons in the bacterial genome [6], [7], [8]. Initially designed for $E$. coli, these ITS regions have seven copies (seven loci) in the bacterial DNA. These non-coding regions accumulate more mutations due to lack of selective pressures, which leads to more variability between bacterial strains. In the known, sequenced $E$. coli DNA, these regions are known to vary both from locus to locus in a single genome, and between the genomes of different strains.

In this paper, we present an in-silico study to investigate the sensitivity of the pyroprinting method. Given a collection of possible DNA sequences that can be found in the sequenced ITS regions, we construct a collection of all possible theoretical combinations. Each such combination represents a theoretically possible strain of $E$. coli. We construct a pyroprint model of each strain, and then build a matrix of pairwise similarities between the pyroprints.

In earlier work [5] we described an in vitro study involving collection of fecal samples, isolation of $E$. coli from the samples, and subsequent pyroprinting of the obtained isolates.
The pyroprints were then compared to each other, and determination of whether multiple isolates belonged to the same strain was made based on how similar the pyroprints were.

In this work we investigated the distribution of Pearson correlations between pairs of theoretical strains. Theoretical strains yielding a correlation value above $99 \%$ are considered hard to differentiate. Over two million theoretical E. coli strains were generated using sequence data from the National Center for Biotechnology Research (NCBI). We used CUDA to speed up the computation of over two trillion Pearson correlations based on the work by Kijsipongse [9] and Chang [10].

\section{IN SILICO STUDY}

The goal of our in silico study is to simulate a wide range of possible isolates using a collection of known alleles, construct their theoretical pyroprints and generate Pearson correlation coefficients for each pair of constructed pyroprints. This section provides a brief outline of the study.

In the context of our simulation study, an allele is a single unique DNA sequence that can be found in the DNA region being pyrosequenced. In $E$. coli, the ITS regions $23 \mathrm{~S}-5 \mathrm{~S}$ and $16 \mathrm{~S}-23 \mathrm{~S}$ are the pyroprinting targets - each have seven loci in the genome. Additionally, a theoretical isolate ${ }^{1}$ is any collection of seven alleles from the 23S-5S ITS region. The same allele may be present multiple times in the isolate. The combination describes the DNA material subject to in silico pyroprinting. Essentially, a combination represents a group of $E$. coli strains that cannot be distinguished via in silico pyroprinting.

a) Input: The input to the study is a collection of 24 alleles for the $23 \mathrm{~S}-5 \mathrm{~S}$ ITS region to be used for construction of different combinations. These alleles have been "harvested" from the NCBI genome database which contains complete genomes for 36 strains of $E$. coli. An additional input to the study is a specific dispensation sequence used to construct all pyrosequences and pyroprints in the study.

Table I shows the total number of theoretical isolates generated for several sizes of the allele collection used in our study. Given $m$ different alleles, the total number $N$ of possible theoretical isolates is the number of unordered combinations of seven alleles selected with replacement:

\footnotetext{
${ }^{1}$ Also referred to as an isolate or combination
} 


\begin{tabular}{|l|l|l|}
\hline \# of Alleles & \# of Isolates & \# of comparisons \\
\hline \hline 10 & 11,440 & $65,431,080$ \\
\hline 11 & 19,448 & $189,102,628$ \\
\hline 12 & 31,824 & $506,367,576$ \\
\hline 13 & 50,388 & $1,269,450,078$ \\
\hline 14 & 77,520 & $3,004,636,440$ \\
\hline 24 & $2,035,800$ & $2,072,239,802,100$ \\
\hline
\end{tabular}

TABLE I

DATASET SIZES GIVEN NUMBER OF ALLELES

\begin{tabular}{|l|l|l|}
\hline Implementation & Time in kernel calls (s) & Time in host (s) \\
\hline \hline Initial & 4.0263 & 0.1111 \\
\hline Improved(Global) & 16.5592 & 0.0786 \\
\hline Improved(Constant) & 8.0397 & 0.0746 \\
\hline
\end{tabular}

TABLE II

AMOUNT OF TIME SPENT IN DEVICE VS HOST FOR RUNS WITH 10 ALLELES

$$
N=\frac{(m+6) !}{7 !(m-1) !}
$$

b) Procedure: The study proceeds in four steps:

1) Isolate generation: Generate every possible combination of seven alleles (unordered, with replacement). Each combination represents a single unique theoretical isolate in our study.

2) Pyroprint generation: For each isolate, construct its pyroprint:

a) Pyrosequence each individual allele of the isolate.

b) Combine individual pyrosequences into a pyroprint by adding up the pyrosequence vectors.

3) Pearson correlation coefficient computation: for each pair of pyroprints, compute the Pearson correlation between them.

4) Histogram generation: separate all computed Pearson correlations coefficients into a number of "buckets" based on their values.

c) Output: The output of the study is the histogram of Pearson correlation coefficient values. The histogram is constructed and reported with respect to a specific collection of buckets, which can be considered a third input parameter to the process. For each bucket, represented by the low and high value, the raw count of Pearson correlations that fall between low and high is to be computed and reported.

\section{EVALUATION}

For our evaluation, we used a machine running Arch Linux 3.3.7-1 that has an Intel Q8200 (Quad Core 2.33GHz) processor, 4GB of DDR3 RAM, and a GTX 260 (192 cores, 896

\begin{tabular}{|l|l|l|l|}
\hline Implementation & occupancy & registers/thread & \# kernel calls \\
\hline \hline Initial & 1 & 12 & 276 \\
\hline Improved(Global) & 0.5 & 23 & 276 \\
\hline Improved(Constant) & 0.5 & 23 & 276 \\
\hline
\end{tabular}

TABLE III

PRofiling Details For RUNS With 10 ALLELES

\begin{tabular}{|l|l|}
\hline Correlation Value Range & \% of comparisons \\
\hline \hline $0-99 \%$ & $99.69 \%$ \\
\hline $99-99.5 \%$ & $0.275 \%$ \\
\hline $99.5-99.7 \%$ & $0.0263 \%$ \\
\hline $99.7-100 \%$ & $0.00873 \%$ \\
\hline
\end{tabular}

TABLE IV

PERCENTAGE OF COMPARISON VALUES FALLING WITHIN A SPECIFIED RANGe of POSSIBLe PEARSON CORRELATION COEFFicient VAlues

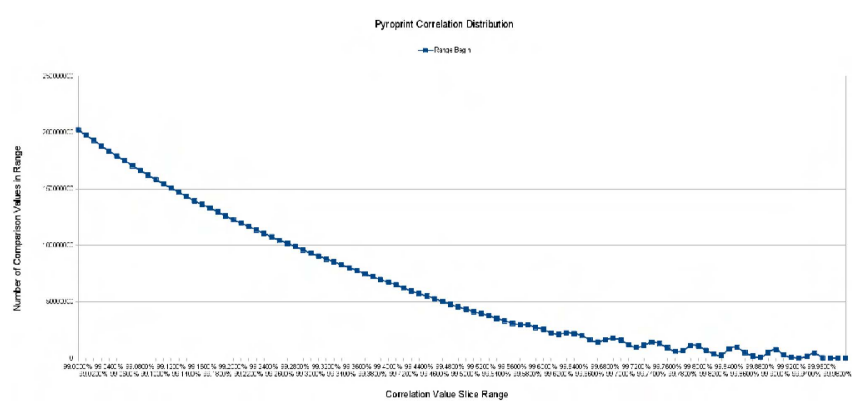

Fig. 1. Distribution of Pyroprint Correlations for 24 alleles

MB of RAM, and compute capability 1.3). Our simulation was developed using pyCUDA and was tested using CUDA toolkit 4.2 (python2-pycuda and cuda-toolkit packages). At times we had a Tesla2070 available for use, however we were only able to get a limited number of runs and are not fully confident in the results observed and so did not include the results in this paper.

For all runs, our input dataset was a set of reference $E$. coli DNA sequences from the $16 \mathrm{~s}-23 \mathrm{~s}$ ITS region. We were given the primer TTGGATCAC for pyrosequencing as well as the dispensation sequence, AACACGCGA23 (GATC) GAA. To simulate an $E$. coli isolate we selected, and combined, seven alleles from the set of alleles extracted during pyrosequencing. A pyroprint for each isolate was then constructed as a sum of the seven alleles chosen for the isolate. Given these parameters, our simulation extracted a set of 24 alleles from the reference $E$. coli DNA sequences. As depicted in Table I, 24 alleles yields over 2 million unique isolates, of which there are over 2 trillion pairwise correlations to be computed. For reference, Table I shows how many isolates and comparisons were made given a set number of alleles.

To assess the improvement of our implementation we compare our initial implementation to two versions of a subsequent implementation which use global and constant memory. We refer to our initial implementation as version 0 , and constant memory and global memory versions of our new implementation as version $1_{c}$ and $1_{g}$, respectively. For each version we observed five runs of five different numbers of alleles $(10-14)$. Each run was executed using $16 \times 16$ thread-block dimensions and $32 \times 32$ grid-block dimensions.

The runtime difference between version 0 and version $1_{c}$ is less than the difference between version $1_{g}$ and $1_{c}$, as seen in Table V. While this makes it seem that our use of constant memory contributes more to our runtime improvement than 


\begin{tabular}{|l|l|l|l|l|l|l|}
\hline Implementation & 10 & 11 & 12 & 13 & 14 & 24 \\
\hline \hline Initial & 19.1507 & 52.2426 & 138.8790 & 341.9866 & 813.8992 & Not included \\
\hline Improved(Global) & 17.4501 & 44.6382 & 110.5517 & 277.4986 & 621.5918 & 122318.7881 \\
\hline Improved(Constant) & 8.8498 & 23.5151 & 62.6434 & 156.7317 & 392.7217 & 104651.4704 \\
\hline
\end{tabular}

TABLE V

RUN TIMES FOR $10,11,12,13,14$, AND 24 ALLELES IN SECONDS

our modification of the isolate generation method, this is not exactly the case.

The performance difference between version 0 and version $1_{c}$ is much smaller using a Tesla2070 than it is using a GTX260. The reason for this is that the GTX260 is much more memory constrained. It has only $896 \mathrm{MB}$ of global memory available where a Tesla2070 has up to 6GB of global memory available. Each version of our implementation must copy data into global memory on device, so the more memory available, the less times data will have to be copied across the PCI-e bus to device. However, version $1_{c}$ (and version $1_{g}$ ) amortizes this cost by only requiring a single data transfer to device. This is particularly emphasized by the fact that the runtime of the pearson correlation kernel for version $1_{c}$ is $\approx 29.9 \mathrm{~ms}$ whereas it is only $\approx 15.3 \mathrm{~ms}$ for version 0 . These results suggest that our performance increase is primarily in memory and bus usage.

The distribution of Pearson correlation coefficient values, depicted in Figure 1, shows that the amount of comparison values above $99 \%$ correlation value significantly drops off for higher correlation values. This distribution can also be seen in Table IV. For our simulation, over $99.6 \%$ of all correlation coefficients fall below $99 \%$. In preliminary studies conducted on actual pyroprints, multiple pyroprints of the same isolate showed correlations above $99.7 \%$. However table IV shows that only $0.00873 \%$ of correlations fall into that category. This suggests that the pyroprinting methodology is sufficiently sensitive to distinguish different bacterial strains.

\section{CONCLUSION}

Despite the drawbacks of our new implementation, its scalability is far beyond our initial implementation. Our new implmementation requires minimal preparation for the GPU to begin computation: 24 alleles $\times 104$ bytes $(1$ byte per pyroprint index), boundary contraints (number of unique isolates), and the range of isolates to compare. This means that the kernel will be able to compute all comparisons once preparation for the kernel has been made without the need for additional data transfers. Our initial implementation may have a fast kernel, but memory constraints prevent it from being scalable and portable.

\section{FUTURE WORK}

Work is being done to implement our simulation with multiGPUs in a single machine as well as using open MPI to be distributed across many machines. Although this data shows the most significant advantage our new implementation has over our initial implementation, due to time and availability constraints, the appropriate modifications could not be made to our simulation to be included here.

\section{ACKNOWLEDGMENT}

This work was supported in part by an undergraduate education grant from the W.M. Keck foundation.

\section{REFERENCES}

[1] T. M. Scott, J. B. Rose, T. M. Jenkins, S. R. Farrah, and J. Lukasik, "Microbial source tracking: Current methodology and future directions," Appl. Environ. Microbiol., vol. 68, pp. 5796 - 5803, dec 2002.

[2] J. M. Simpson, J. W. S. Domingo, and D. J. Reasoner, "Microbial source tracking: State of the science," Environmental Science and Technology, vol. 36, pp. $5279-5288$, dec 2002.

[3] T. R. Desmarais, H. M. Solo-Gabriele, and C. J. Palmer, "Influence of soil on fecal indicator organisms in a tidally influenced subtropical environment," Applied and Environmental Microbiology, vol. 68, pp. 1165 - 1172, mar 2002.

[4] D. M. Gordon, "Strain typing and the ecological structure of Escherichia coli," Journal of AOAC International, vol. 93, pp. 974 - 984, may 2010.

[5] A. Montana, A. Dekhtyar, E. Neal, M. Black, and C. Kitts, "Chronologysensitive hierarchical clustering of pyrosequenced dna samples of e. coli: A case study," in 2011 International Conference on Bioinformatics and Biomedicine. IEEE, 2011, pp. 155-159. [Online]. Available: http://www.computer.org/portal/web/csdl/doi/10.1109/BIBM.2011.99

[6] S. L. Boyer, V. R. Flechtner, and J. R. Johansen, "Is the 16s-23s rrna internal transcribed spacer region a good tool for use in molecular systematics and population genetics? a case study in cyanobacteria," Molecular Biology and Evolution, vol. 18, no. 6, pp. 1057 - 1069, june 2001.

[7] A. Roth, M. Fischer, M. Hamid, S. Michalke, W. Ludwig, and H. Mauch, "Differentiation of phylogenetically related slowly growing mycobacteria based on 16s-23s rrna gene internal transcribed spacer sequences," Journal of Clinical Microbiology, vol. 36, no. 1, pp. 139 - 147, january 1998.

[8] S. Tyler, C. Strathee, K. Rozee, and W. Johnson, "Oligonucleotide primers designed to differentiate pathogenic pseudomonads on the basis of sequencing of genes coding for 16s-23s rrna internal transcribed spacers," Clinical and Diagnostic Laboratory Immunology, vol. 2, no. 4, pp. 448-453, july 1995.

[9] E. Kijsipongse, S. U-Ruekolan, C. Ngamphiw, and S. Tongsima, "Efficient large pearson correlation matrix computing using hybrid $\mathrm{mpi} / \mathrm{cuda}$," in Computer Science and Software Engineering (JCSSE), 2011 Eighth International Joint Conference on, may 2011, pp. 237 241.

[10] Dar-Jen Chang, A. H. Desoky, M. Ouyang, and E. C. Rouchka, "Compute pairwise manhattan distance and pearson correlation coefficient of data points with gpu," in Proceedings of the 2009 10th ACIS International Conference on Software Engineering, Artificial Intelligences, Networking and Parallel/Distributed Computing, ser. SNPD '09. Washington, DC, USA: IEEE Computer Society, 2009, pp. 501-506. [Online]. Available: http://dx.doi.org/10.1109/SNPD.2009.34 